\title{
NON-STATIONARY CONDITION MONITORING THROUGH EVENT ALIGNMENT
}

\author{
Niels Henrik Pontoppidan and Jan Larsen \\ Informatics and Mathematical Modeling, Building 321 \\ Technical University of Denmark, DK-2800 Lyngby, Denmark \\ Phone: +45 4525 3899,3923 - Fax: +45 45872599 \\ E-mail: nhp,jl@imm.dtu.dk - Web: isp.imm.dtu.dk
}

\begin{abstract}
We present an event alignment framework which enables change detection in non-stationary signals. change detection. Classical condition monitoring frameworks have been restrained to laboratory settings with stationary operating conditions, which are not resembling real world operation. In this paper we apply the technique for non-stationary condition monitoring of large diesel engines based on acoustical emission sensor signals. The performance of the event alignment is analyzed in an unsupervised probabilistic detection framework based on outlier detection with either Principal Component Analysis or Gaussian Processes modeling. We are especially interested in the true performance of the condition monitoring performance with mixed aligned and unaligned data, e.g. detection of fault condition of unaligned examples versus false alarms of aligned normal condition data. Further, we expect that the non-stationary model can be used for wear trending due to longer and continuous monitoring across operating condition changes.
\end{abstract}

\section{INTRODUCTION}

We pursue Condition Monitoring (CM) systems which are capable of detecting faults in large diesel engines used for propulsion and power generation. Such operation involves frequent changes in either load or speed. The current problem is that those trivial changes result in false alarms that cannot be separated from alarms originating from real faults.

MAN B\&W Diesel has conducted experiments simulating realistic marine operation with multiple loads. Faults resembling scuffing was induced by means of shutting the lubricating oil system off after the engine was started Scuffing is a severe fault that with time evolves into damaging contact between cylinder piston and liner. In the acquired data set we have have identi- 


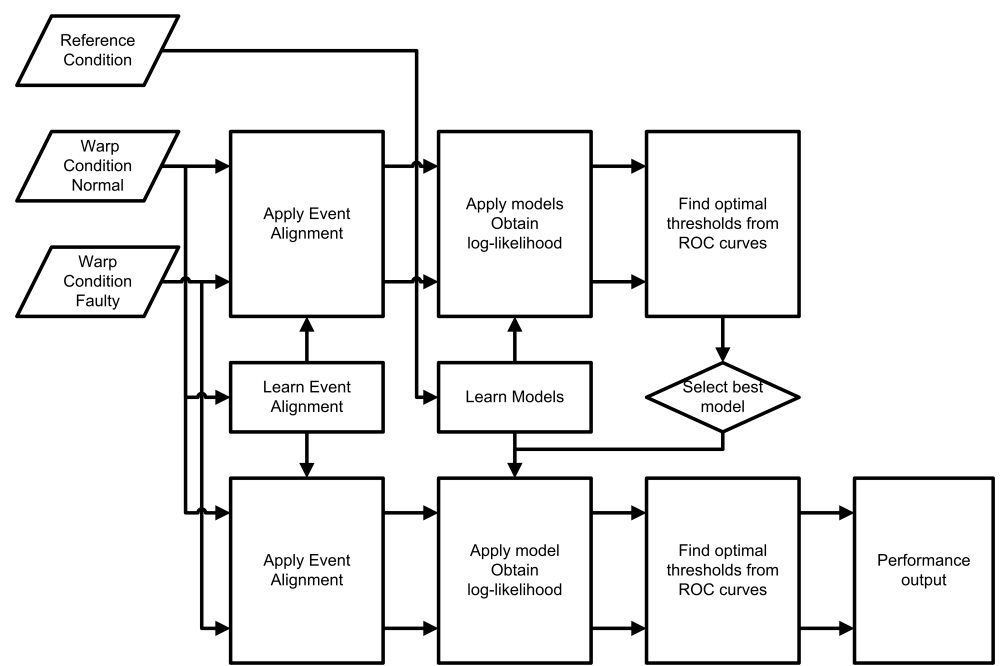

Figure 1: System overview. The figure outlines the flow of information during the experiments.

fied a stable functional dependency between signals from different operational conditions. We propose a novel method that builds invariance into the Condition Monitoring System (CMS) by inverting those functional changes prior to outlier detection. The traditional approach of sampling of data in the crank angular domain [1] is not enough to remove those changes. Further the available Dynamic Time Warp algorithm [3, 9] was discarded since repeated time frames produced fault-like signals.

We have previously cast unsupervised condition monitoring as an outlier detection problem with generative models [10]. The generative models allows for localization of large deviations that indicates the origin of the fault. With this setup we have successfully detected induced scuffing (piston rubbing against the liner) and externally generated faults under stationary conditions. However, we were not able to distinguish between alarms due to faults or operational changes under non-stationary conditions. Also, other recent monitoring applications $[2,5]$ have been limited to fixed operating conditions.

\section{MODELING}

The following section presents the data setup and the describe the use of Principal Component Analysis and Gaussian Processes for modeling in a condition monitoring framework.

Data setup. Data was acquired on MAN B\&W Diesel's two-stroke test bed engine, under controlled varying conditions. The acquired ultrasonic acoustic emission signals were preprocessed by short time root mean square and converted from time domain into the crank angular domain using a crank 

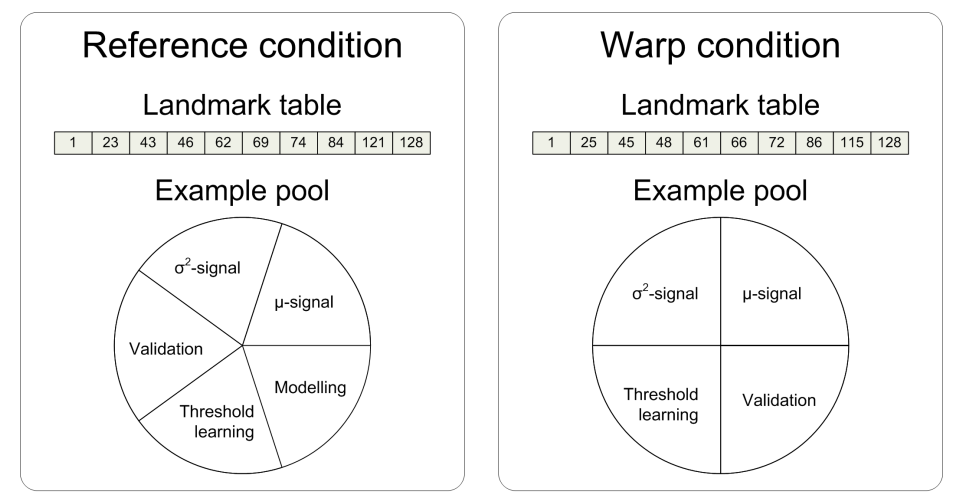

Figure 2: Data partitioning for Event alignment and Change detection. Different sizes of partions are allowed. We use different sets of data to learn the warping parameters ( $\mu$ and $\sigma^{2}$-signals), rejection threshold. Further we also learn the model parameters from a set of data from the reference condition. Finally we use unused examples for validation of the performance.

angle tachometer. Further the signals were downsampled to enable Gaussian Process modeling. The properties of each RMS AE signal, $\boldsymbol{x}$, is: $d$ nonnegative elements sampled at specific angular positions at constant angular sample rate regardless of engine operating conditions. Figure 1 show the system overview explained in this section. We center data (denoted $\tilde{\boldsymbol{x}}$ ) before modeling by subtracting the mean obtained across a subset of Normal Condition (NC) examples. Throughout the experiments we estimate parameters in a step-wise manner using resampling. As shown in Figure 2 a specific data subset used to learn a parameter is only used one time. Two modeling schemes for the analysis of the performance of the event alignment are deployed. The approach is a mixture of supervised and unsupervised learning. Unsupervised modeling is used to model NC data by training a set of parameters $\boldsymbol{\theta}$ in Principal Component Analysis (PCA) and Gaussian Process (GP) models described below. The log-likelihood of the $\mathrm{NC}$ model is used as a measure of how much an example belongs to a model, and the log-likelihood density of NC and Faulty Condition (FC) examples in general separates. A rejection threshold is obtained in a supervised manner by finding selecting an optimal point on the Receiver Operator Characteristics Curve from a set of labeled NC and FC examples.

Principal Component Analysis Model. From a set of $N$ centered normal examples $\tilde{\boldsymbol{x}}$ (size $d \times 1$ ) we build the training matrix $\boldsymbol{X}_{\mathcal{T}}$ by stacking $($ size $d \times N) . \boldsymbol{X}_{\mathcal{T}}=\left[\tilde{\boldsymbol{x}}_{1}, \tilde{\boldsymbol{x}}_{2}, \cdots, \tilde{\boldsymbol{x}}_{N}\right]$. From this training matrix we estimate a principal component matrix $\boldsymbol{P C}$ (size $N \times N)$ ), and a projection matrix $\boldsymbol{U}$ $($ size $d \times N)$ through the Singular Value Decomposition (SVD) $\boldsymbol{X}_{\mathcal{T}}=\boldsymbol{U} \boldsymbol{\Lambda} \boldsymbol{V}^{\top}$. The number of principal components $k$ is controlled by using the first $k$ columns of $\boldsymbol{U}$ and $(k)$ rows of $\boldsymbol{P C}$.

$$
\boldsymbol{P C}=\boldsymbol{\Lambda} \boldsymbol{V}^{\top}
$$



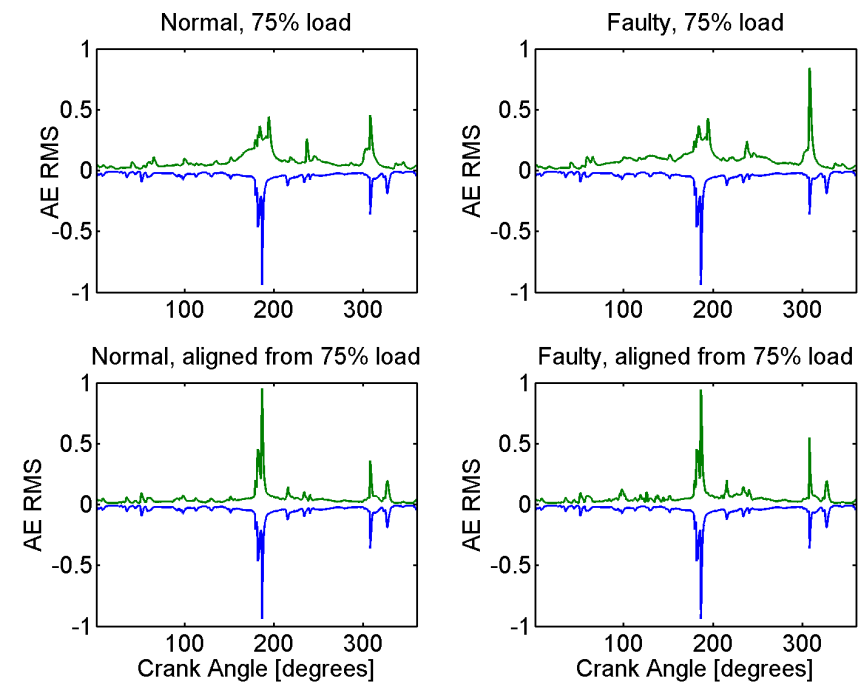

Figure 3: Application of the event alignment. The normal condition signal is displayed with negative sign. Normal and faulty condition $75 \%$ load data were event aligned using the warp for normal condition $75 \%$ load data. Since the faulty condition $75 \%$ load does not comply with this model, the aligned faulty condition examples display deviation around 100-150 degrees.

When applying the PCA model to new examples we multiply with the first $k$ transposed columns of $\boldsymbol{U}$ from the left and obtain $\boldsymbol{s}_{k}$ plus the noise $\boldsymbol{\epsilon}$, as the remaining $d-k$ components that span a Gaussian noise space $[8,10]$.

$$
\begin{aligned}
\boldsymbol{s}_{k} & =\boldsymbol{U}_{k}^{\top} \tilde{\boldsymbol{x}} \\
\tilde{\boldsymbol{x}} & =\boldsymbol{U}_{k} \boldsymbol{s}_{k}+\boldsymbol{\epsilon}
\end{aligned}
$$

It follows directly from the properties of the SVD and (2) that the principal components of $\mathrm{NC}$ examples follow a multivariate zero mean Gaussian with covariance $\boldsymbol{\Lambda}_{k}$ (using the first $k$ columns and rows of $\boldsymbol{\Lambda}$ ). Let $\boldsymbol{\theta}$ denote all estimated parameters $[8,10]$, then $p(\boldsymbol{x} \mid \boldsymbol{\theta}, k)=p\left(\boldsymbol{s}_{k} \mid \boldsymbol{\theta}\right) p(\boldsymbol{\epsilon} \mid \boldsymbol{\theta})$ and the loglikelihood is $\mathcal{L}=\log p\left(\boldsymbol{s}_{k} \mid \boldsymbol{\theta}\right)+\log p(\boldsymbol{\epsilon} \mid \boldsymbol{\theta})$

Gaussian Process Model. As an alternative to the PCA subspace model we can perform modeling directly in the observed domain. Through Gaussian Process (GP) modeling we obtain a measure of how much an example deviates from the reference condition. From Gibbs and MacKay [7] we have (with interchanged $\boldsymbol{t}$ and $\boldsymbol{x}$ relative to Gibbs MacKay notation)

$$
\begin{aligned}
Q\left(t_{i}, t_{j}\right) & =\theta_{2}^{2} \exp \left(-\frac{\left(t_{i}-t_{j}\right)^{2}}{2 \theta_{1}^{2}}\right)+\theta_{3}^{2} \delta\left(t_{i}, t_{j}\right) \\
p(\tilde{\boldsymbol{x}} \mid \mathbf{Q}, \mathbf{t}) & =\frac{1}{Z} \exp \left\{-\frac{1}{2} \tilde{\boldsymbol{x}}^{\top} \mathbf{Q}^{-1} \tilde{\boldsymbol{x}}\right\},
\end{aligned}
$$




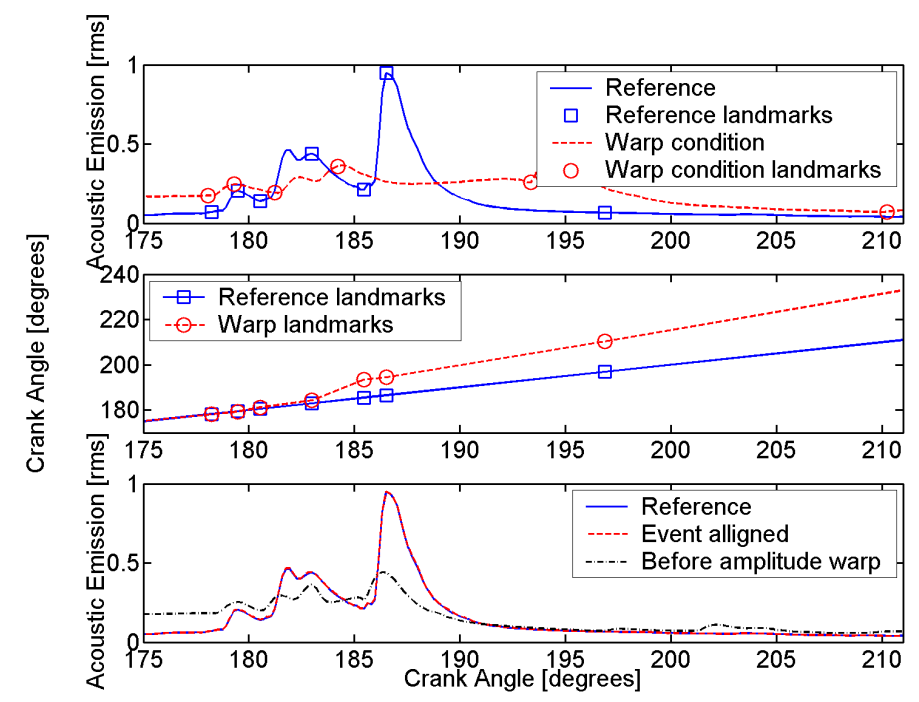

Figure 4: Examples and landmarks during injection period. The upper figure displays the mean signals and landmarks for the two conditions. The middle figure display the landmarks. In the beginning they are almost equal and the warp condition evolves slower than the reference condition. The lower figure display the mean signal before amplitude warp, and the mean signal of the fully event aligned. The mean signals are shown before, during and after event alignment. Notice that the event alignment results in equal mean signals.

where $\mathbf{t}$ is the vector of crank positions, and $\tilde{\boldsymbol{x}}$ is the corresponding observed centered values. The covariance matrix $Q$ is a function of the index vector $\boldsymbol{t}$ and the parameters $\boldsymbol{\theta}$. The last term of Equation 3 is the noise part. The negative log-likelihood for the example $\tilde{\boldsymbol{x}}$ given the parameters $\boldsymbol{t h e t a}$ defining the covariance matrix $\boldsymbol{Q}$ is

$$
\mathcal{L}=\frac{1}{2} \log |\mathbf{Q}|+\frac{1}{2} \tilde{\boldsymbol{x}}^{\top} \mathbf{Q}^{-1} \tilde{\boldsymbol{x}}
$$

For each training examples $\tilde{\boldsymbol{x}}_{n_{\theta}}, n_{\theta} \in N_{\theta}$ we train an independent GP with parameters $\left\{\boldsymbol{\theta}_{n_{\theta}}\right\}$ through minimization of $\mathcal{L}$ using minimize.m [11]. Finally we perform average over the parameters obtained from different training examples to obtain the final model parameters.

$$
\begin{aligned}
\widehat{\boldsymbol{\theta}}_{n_{\theta}} & =\arg \min _{\theta}\left\{\frac{1}{2} \log |\mathbf{Q}|+\frac{1}{2} \tilde{\boldsymbol{x}}_{n_{\theta}}^{\top} \mathbf{Q}^{-1} \tilde{\boldsymbol{x}}_{n_{\theta}}\right\} \\
\widehat{\boldsymbol{\theta}} & =\log \left(\frac{1}{\operatorname{card}\left(N_{\theta}\right)} \sum_{n_{\theta} \in N_{\theta}} \exp \left(\widehat{\boldsymbol{\theta}}_{n_{\theta}}\right)\right)
\end{aligned}
$$

In order to ensure positive parameters without enforcing constraints minimize.m uses reparameterization, hence, the averaging takes place in the natural parameter space and explains the exp and log in (7). The original implementation of the Gaussian Processes was due to Carl Rasmussen [11], but the we 
have customized the input/output structure to fulfill our needs, e.g., allowing the training and use of the $\boldsymbol{Q}$ matrix.

\section{DETECTION}

Outlier detection with log-likelihood is based that NC and FC examples separate in log-likelihood space. For instance we expect that the number and/or characteristics of the underlying hidden sources are changed when entering the FC, thus examples acquired from a FC are poorly described by a model trained on $\mathrm{NC}$ examples. We expect that combinations of increased noise or increased strength of certain acoustical sources results in a lowver loglikelihood value. ¿From a set of labeled preprocessed examples we build the accumulated densities for the features $p\left(\mathcal{L}_{\mathrm{NC}} \leq \tau\right)$ and $p\left(\mathcal{L}_{\mathrm{FC}} \leq \tau\right)$. Each value of $\tau$ corresponds to a true detection / false alarm ratio, and we choose the optimal rejection threshold $\hat{\tau}$ that is closest in distance to $100 \%$ detection and $0 \%$ false alarms. The threshold can also be obtained in other manners, e.g., selecting the threshold that detects most faults with a constant false alarm rate.

\section{EVENT ALIGNMENT (WARPING)}

We present event alignment as a novel tool for non-stationary Condition Monitoring (CM) of large marine diesel engines. The tool is necessary since the current CMSs are not invariant to certain known operational changes in particular load/speed changes. Given two different NC, the event alignment transforms examples from one condition into the other condition, thus facilitating a CMS trained on the reference system to correctly detect deviations under both conditions. The result is that the CMS becomes invariant to changes between the two NC's. With more NC's we expect that interpolation between a few warps is possible. Non-stationary condition monitoring is important when considering diesel engines since the operating conditions change frequently. Under normal marine conditions Frances et al. [6] have observed large variability. In our data sets we have found that such variability is largely described by the changing operation conditions, indicating that unwanted false alarms could be suppressed by adopting to the changes invoked by the operating conditions. It should also be noted that application of the same model on a continuous flow of data could allow for trending of wear, that is not necessarily possible with multiple models, as models might focus on different properties of the condition modes.

Obviously one should take care that examples which do not originate from the warp condition are not transformed into the reference condition. For instance the event alignment should try to preserve the same variations as in the reference condition, as this prevents the event aligned examples of becoming super-normal. Furthermore it prevents examples from other 
conditions of being transformed into the reference condition. Overfitting with event alignment is still an open issue which needs further research.

Dynamic Time Warp based on phase vocoder techniques [4] described by Ellis [3] and Keough [9] performs the time-warp while keeping the frequency content unchanged. The phase vocoder is based on short time Fourier transformation and accomplishes the time-warp by interchanging the number of samples between overlapping time frames at playback time, e.g., moving the overlapping windows further apart in order to stretch the signal. Dynamic Time Warping is uninteresting for CM in the time domain, as it repeats or drops time frames if necessary, possible duplicating peaks or removing fault signatures. Instead we decide on time-warps that keep the waveform structure or envelope unaltered, e.g. spline interpolation. Also piece-wise linear interpolation was tried, but in the present case the cubic splines provided better results.

\section{Event alignment model}

The event alignment consists of two non-linear warps, the first performs timealignment and the second performs amplitude mapping. Definitions (also see Figure 2)

$\boldsymbol{L}$ The landmark vector defines the angular position of the important events. Most events are described by three landmarks: begin, peak and end.

$\boldsymbol{\mu}$ the vector containing the mean signal (across examples for each angular position)

$\sigma^{2}$ the vector containing the variation around $\boldsymbol{\mu}$.

The landmarks were picked by hand, and are very specific for the application. Even changing a sensor position would change the landmarks. Thus, automatic identification of landmarks is to be addressed in future studies. The event alignment transforms warp condition examples $\boldsymbol{x}_{W}$ described by $\boldsymbol{\mu}_{W}, \boldsymbol{\sigma}_{W}^{2}$ and landmarks $\boldsymbol{L}_{W}$ into aligned examples $\boldsymbol{x}_{A}$ resembling the reference condition $\boldsymbol{\mu}_{R}, \boldsymbol{\sigma}_{R}^{2}$ and $\boldsymbol{L}_{R} . f(\cdot)$ is an interpolating function that performs the time alignment of events based on the two set of landmarks. The vector $\boldsymbol{g}$ is a sample-wise constrained re-scaling factor that accounts for compression of variance when the variance in the warp condition is larger than in the reference condition.

$$
\begin{aligned}
\boldsymbol{x}_{A} & =\left(f\left(\boldsymbol{x}_{W}, \boldsymbol{L}_{R}, \boldsymbol{L}_{W}\right)-\boldsymbol{\mu}_{W}\right) \cdot * \boldsymbol{g}+\boldsymbol{\mu}_{R}, \\
\boldsymbol{\mu}_{W} & =\left\langle f\left(\boldsymbol{x}_{W}, \boldsymbol{L}_{R}, \boldsymbol{L}_{W}\right)\right\rangle \\
g_{i} & =\left\{\begin{array}{ll}
1 & , \sigma_{i R}>\sigma_{W i} \\
\sigma_{R i} / \sigma_{W i} & , \sigma_{R i}>\sigma_{W i}
\end{array}, i=1,2, \ldots, d\right.
\end{aligned}
$$

where $*$ denoting Hadamard matrix multiplication. The constraint prevents amplification of measurement noise. Unconstrained re-scaling can lead to negative values that do not correspond to the non-negative RMS signals. 
In some cases this constraint leads to overfitting, as the aligned examples become "more" normal that the un-aligned examples. In the following section we encounter this problem in experiment 5 for Gaussian Process modeling.

\section{EXPERIMENTS AND RESULTS}

We create pseudo-realistic data sets in order to compensate for lack of data by resampling of examples within periods of stable conditions. Examples are resampled by drawing random examples from pools of data and only used once. That is, examples used to learn the model, warp or threshold are not used during performance evaluation. Resampling of examples facilitates evaluation and analysis of the models at the expense that condition changes become more abrupt, thus analysis of alarm time and trending is not possible.

We measure the performance of event alignment on its ability to correctly separate FC and NC examples during changing operational conditions.

All experiments (see Table 1) where conducted using a model trained on random examples drawn from the $25 \%$ load NC. Table 2 reports the obtained detection rates using the two different modeling schemes. For PCA only the performance with the optimal number of components is reported. Experiment 1 shows the performance of the stationary system on stationary data. Applying the stationary system to non-stationary data would label all normal conditions as faulty since the CMS cannot discriminate between normal variations and true faults, thus the resembling the non-stationary conditions is indeed promising.

Experiment 2 and 3 demonstrate the ability to align other $\mathrm{NC}$ with the reference condition while the event alignment of FC examples using the same model are correctly labeled as faulty. In experiment 2 we obtain the same performance as the stationary system, but in experiment 3 the performance is degraded. This is due to the downsampling of examples. The original AE RMS vectors had $d=2048$ samples per revolution, and since the training of the Gaussian Process model involves inversion of $d \times d$ square matrix, all examples have been downsampled with a factor 8 . The fault leads to unstable timing of events and the downsampling smears out these changes. We notice that the PCA preprocessing suffers more from downsampling than the GP model, however, without any downsampling, PCA also yields 80-90\% detection rate and $15-20 \%$ false alarm rate (similar to the GP with downsampled data). As expected, the overall performance is reduced in comparison with the stationary experiment.

Experiment 4 demonstrates how the non-stationary system is able to discriminate between aligned $\mathrm{NC}$ data and un-aligned FC data. The result is similar to that of the stationary system.

In experiment 5 we test the CMS w.r.t. overfitting. We cheat the system and take examples warped into the normal condition as normal and unwarped NC data as "faulty". The overfitting in the event alignment, i.e., the examples are warped into being super-normal, is detected with the GP 


\begin{tabular}{|c|ll|}
\hline Exp & Normal data & Faulty data \\
\hline 1 & $25 \%$ load, lube oil on & $25 \%$ load, lube oil off \\
2 & $75 \%$ load, lube oil on & $75 \%$ load, lube oil off \\
3 & $50 \%$ load, lube oil "on" & $50 \%$ load, unstable speed \\
4 & $75 \%$ load, lube oil on & $25 \%$ load, lube oil off \\
5 & $50 \%$ load, lube oil "on" & $25 \%$ load, lube oil on \\
6 & Mixed loads, lube oil on & Mixed loads, lube oil off \\
\hline
\end{tabular}

TABle 1: List of experiments. During experiment 3 and 5 examples acquired without lube oil was warped into the reference condition with lube oil.

\begin{tabular}{|c|rcr|ccr|}
\hline Exp & PCA & Detec/False & \# Comp & GP & Detec/False \\
\hline 1 & & $95 /$ & 5 & 2 & $95 /$ & 5 \\
2 & & $95 /$ & 5 & 2 & $95 /$ & 5 \\
3 & & $60-65 / 20-30$ & 3 & $80-90 / 15-20$ \\
4 & & $95 /$ & 5 & 2 & $95 /$ & 5 \\
5 & & $50-60 / 65-80$ & 36 & $80-95 /$ & $0-15$ \\
6 & & $95 /$ & 5 & 2 & $95 /$ & 5 \\
\hline
\end{tabular}

TABle 2: Condition Monitoring Performance. Detec/False denotes detection versus false alarms rate in percentage.

modeling, that incorrectly label 80-95\% of the NC examples as FC. However, using PCA modeling the overfitting disappears, even though the PCA uses much more components than usually, indicating that it is looking for very small changes.

In experiment 6 we collect both aligned and un-aligned examples from experiment 1,2 and 4 in order to demonstrate that the system is capable of performing non-stationary condition monitoring with the same performance as in the individual experiments. This demonstrates that the obtained optimal rejection thresholds are stable in the three experiments 1,2 , and 4 .

\section{CONCLUSION}

The experiments show that non-stationary condition monitoring is indeed possible. It is important to notice that the event alignment does not decrease the overall condition monitoring performance as the results obtained in experiment 2 and 4 are equal to the stationary results in experiment 1 . Furthermore, the performance obtained using both mixed aligned and original data in experiment 6 is the same as in the individual experiments, indicating that the optimal rejection thresholds are fairly constant even with several warp conditions each having its own set of event alignment parameters. The conclusion is that non-stationary CM indeed can be obtained by extending a stationary CMS with event alignment.

Future work will concentrate on refining the method to handle a larger range operation conditions, automatic detection of landmarks, and further 
investigations related to overfitting. In addition, we will evaluate whether the framework will allow for wear trending, which of course calls for new experiments involving much larger time scales. We will also pursue fast Gaussian Processes in order to avoid signal downsampling.

\section{ACKNOWLEDGEMENTS}

The work is supported by EU Competitive and Sustainable Growth Programme GRD2-2001-50014 through the AE-WATT project. Data was provided by MAN B\&W Diesel A/S. Discussions with Sigurdur Sigurdsson and Mads Dyrholm as well as project partners at Heriot-Watt University were very much appreciated.

\section{REFERENCES}

[1] G. Chandroth and A. Sharkey, "Utilising the rotational motion of machinery in a high resolution data acquisition system," in Proc of Computers and ships, May 1999.

[2] G. Chandroth, A. Sharkey and N. Sharkey, "Vibration signatures, wavelets and principal components analysis in diesel engine diagnostics," in Marine Technology ODRA 99, Oct. 1999.

[3] D. Ellis, "Dynamic Time Warp (DTW) in Matlab," Internet, http:// labrosa.ee.columbia.edu/matlab/dtw/.

[4] J. Flanagan and R. Golden, "Phase Vocoder," Bell System Technical Journal, pp. 1493-1509, Nov. 1966.

[5] T. Fog, L. Hansen and J. Larsen et al., "On Condition Monitoring of Exhaust Valves in Marine Diesel Engines," in Y. H. Hu, J. Larsen, E. Wilson and S. Douglas (eds.), Proceedings of the NNSP IX, IEEE, Piscataway, New Jersey, 1999, pp. 225-234.

[6] A. Frances, J. Gill, J. Reuben and J. Steel, "A Study of the Variability of Acoustic Emission Signals from a Medium Size Marine Diesel Engine under Service Conditions," in O. P. Shrivastav, B. Al-Najjar and R. B. Rao (eds.), COMADEM 2003, COMADEM International, Växjö, 2003, pp. 503-512.

[7] M. N. Gibbs and D. J. C. MacKay, "Efficient implementation of Gaussian Processes for Interpolation," http://www.inference.phy.cam.ac.yk/mng10/ GP/gpros.ps.gz, 1997.

[8] L. Hansen and J. Larsen, "Unsupervised Learning and Generalization," in Proceedings of the IEEE Int. Conf. on Neural Networks 1996, 1996, vol. 1, pp. 25-30.

[9] E. Keogh, "Exact Indexing of Dynamic Time Warping," in Proceedings of the 28th VLDB Conference, Hong Kong, China, 2002.

[10] N. Pontoppidan and J. Larsen, "Unsupervised Condition Change Detection In Large Diesel Engines," in C. Molina, T. Adali, J. Larsen, M. Van Hulle, S. Douglas and J. Rouat (eds.), 2003 IEEE NNSP Workshop, Piscataway, New Jersey: IEEE Press, Sept. 2003, pp. 565-574.

[11] C. E. Rasmussen, "minimize.m, gps00.m," http://www.kyb.tuebingen.mpg. de/ ${ }^{\sim} \operatorname{carl}, 2001,2003$. 